\title{
ON MAXIMAL REARRANGEMENT INEQUALITIES FOR THE FOURIER TRANSFORM
}

\author{
BY
}

\author{
W. B. JURKAT AND G. SAMPSON ${ }^{1}$
}

\begin{abstract}
Suppose that $w$ is a measurable function on $\mathbf{R}^{n}$ and denote by $W=w^{*}$ the decreasing rearrangement of $|w|$ (provided that it exists). We show that the $n$-dimensional Fourier transform $\hat{f}$ satisfies
\end{abstract}

$$
\|w \cdot \hat{f}\|_{q} \leqslant\left\|W(\hat{f})^{*}\right\|_{q} \leqslant C\left\|W(t) \int_{0}^{1 / t} f^{*}\right\| \quad(C \text { absolute constant }),
$$

if $1<q<x$ and $t^{2 / q^{-1}} W(t) \searrow$ for $t>0$. We also show that

$$
\|w \hat{f}\|_{q} \geqslant c_{n, q}\left\|w(x) \int_{|y| \leqslant 1 /|x|} f(y) d y\right\|_{q} \quad \text { ( } f \text { nonnegative), }
$$

if $1<q<\propto$ and $w$ is nonnegative and symmetrically decreasing. Inequality (2) implies that (1) is maximal in the sense that the left side reaches the right side if $f$ is nonnegative and symmetrically decreasing. Hence, (1) implies all other possible estimates in terms of $W$ and $f^{*}$. The cases $q \neq 2$ of (1) can be derived from the case $q=2$ (and same $f$ ) by a convexity principle which does not involve interpolation. The analogue of ( 1 ) for Fourier series is due to $\mathrm{H}$. L. Montgomery if $q \geqslant 2$ (then the extra condition on $W$ is automatically satisfied).

1. Introduction. We consider the $n$-dimensional $(n \geqslant 1)$ Fourier transform

$$
\hat{f}(x)=\int_{\mathbf{R}^{n}} f(y) e(x \cdot y) d y, \quad e(t)=\exp (2 \pi i t) \quad \text { for } t \in \mathbf{R}
$$

where $x \cdot y$ is the scalar product and $f \in L^{1}\left(\mathbf{R}^{n}\right)$ or $\in L^{2}\left(\mathbf{R}^{n}\right)$ or just a "test function", i.e. smooth and "small" at $\infty$; and we denote by $f^{*}$ the (1-dimensional) decreasing rearrangement of $|f|$ over $(0, \infty)$ which has the property

$$
\left|\left\{x \in \mathbf{R}^{n}:|f(x)| \geqslant s\right\}\right|=\left|\left\{t \in(0, \infty): f^{*}(t) \geqslant s\right\}\right| \text { for } s>0 .
$$

Our objective is to estimate the "local averages"

$$
\int_{E}|\hat{f}|^{2} \quad \text { or } \int_{E}|\hat{f}|^{q} \quad(q \geqslant 2)
$$

in terms of $T=|E|$ and $f^{*}$. More generally, we want to estimate weighted norms, i.e.

$$
\int_{\mathbf{R}^{n}}|w \hat{f}|^{q} \quad(1<q<\infty, w \text { nonnegative and measurable }),
$$

Received by the editors May 25, 1982 and, in revised form, February 28, 1983.

1980 Mathematics Subject Classification. Primary 42A38, 42B10.

'The work of the first author was supported in part by the National Science Foundation. 
in terms of $w^{*}=W$ and $f^{*}=F$. So we are interested in determining the supremum of (1.1) if we vary $w$ and $f$ in such a way that $W$ and $F$ remain the same. An equivalent problem is to estimate

$$
\int_{0}^{\infty}\left(W(\hat{f})^{*}\right)^{q} \quad(1<q<\infty, W \geqslant 0)
$$

in terms of $f^{*}$. If the estimate gives the sup within constant factors we call the estimate maximal.

In this paper we give the maximal estimates for $q \geqslant 2$ and, under a slight restriction on $W$, also those for $1<q \leqslant 2$ (cf. Corollary 4). The corresponding problem for Fourier series was recently discussed by H. L. Montgomery [9] for $q \geqslant 2$. He also showed that the case $q>2$ can be derived from the case $q=2$ by a general principle which does not involve interpolation. Through the introduction of weights we are also able to deal with exponents $1<q \leqslant 2$. We have abstracted the general principle (cf. §5) so that these cases can also be derived from the case $q=2$. The maximal upper estimates are given in $\$ \S 3$ and 6 . We also found very sharp lower estimates for Fourier transforms of positive functions (cf. $\$ \S 4$ and 7). These can, e.g., be used to show that various forms of the upper estimates are maximal. In $\S \S 9$ and 10 we discuss Fourier series and Fourier coefficients thereby adding new results to those of $\mathrm{H}$. L. Montgomery.

As an application of our maximal inequalities we can decide whether or not the Fourier transform maps a given rearrangement invariant Banach space into another given space of that type. A typical case would be an inequality of the form

$$
\left\{\int_{0}^{\infty}\left(W(\hat{f})^{*}\right)^{q}\right\}^{1 / q} \leqslant A\left\{\int_{0}^{\infty}\left(V f^{*}\right)^{p}\right\}^{1 / p} \quad(1<p \leqslant q<\infty),
$$

where $0 \leqslant W \searrow, 0 \leqslant V \nearrow$, and $A$ is a positive finite constant. Such inequalities contain almost all known norm inequalities for the Fourier transform. We are dealing with these questions in a separate paper [6]; cf. also a forthcoming paper of B. Muckenhoupt [10].

2. Notations. Here we collect some notations which will be used throughout the paper: Our functions $f$ are permitted to be complex-valued. We say that $f$ is radial on $\mathbf{R}^{n}$ if $f(x)$ is defined for $x \neq 0$ and depends only on $|x|=\sqrt{x \cdot x}$. It is convenient to write $f(x)=F\left(\gamma_{n}|x|^{n}\right)$, where $F(t)$ is defined for $t>0$ and $\gamma_{n}$ denotes the volume of the $n$-dimensional unit ball. If $f^{*}$ exists (as a finite function) we define the symmetrically decreasing rearrangement by $f^{[*]}(x)=f^{*}\left(\gamma_{n}|x|^{n}\right)$ for $x \neq 0$.

The arithmetic mean operator is defined by

$$
\mathbf{A} f(x)=\frac{1}{\gamma_{n}|x|^{n}} \int_{|v| \leqslant|x|} f(y) d y \quad\left(f \in L_{\mathrm{loc}}\left(\mathbf{R}^{n}\right), x \neq 0\right) .
$$

In the integration it may be convenient to use polar coordinates

$$
y=t \eta \quad \text { with } t>0,|\eta|=1 \text {. }
$$


Let $d \eta$ denote the usual measure on the $(n-1)$-dimensional unit sphere $S^{n-1}$ normalized by $\int_{S^{n-1}} d \eta=1$. Then we can write $d y$ as a product measure $d\left(\gamma_{n} t^{n}\right) d \eta$. Thus we see, e.g., that

$$
\mathbf{A} f^{[*]}(x)=\frac{1}{\gamma_{n}|x|^{n}} \int_{0}^{\gamma_{n}|x|^{n}} f^{*}(t) d t=f^{(*)}\left(\gamma_{n}|x|^{n}\right), \quad x \neq 0
$$

where we use

$$
f^{(*)}(t)=\frac{1}{t} \int_{0}^{t} f^{*}(s) d s, \quad t>0
$$

for the averaged rearrangement.

We use $P \ll Q$ to abbreviate an inequality $|P| \leqslant b|Q|$ with some positive finite constant $b$ (independent of the specified variable quantities), and we use $P \preceq Q$ to indicate $P \ll Q$ and $Q \ll P$. Absolute constants will be denoted by $c, C, \ldots$ and will be indexed if necessary. They are not necessarily the same at each occurrence.

3. The basic local upper estimate. We prove the following "local" version of Bessel's inequality.

THEOREM 1. For $T>0, f \in L^{2}\left(\mathbf{R}^{n}\right)$ we have

$$
\int_{0}^{T} \hat{f}^{*}(t)^{2} d t \ll T\left(\int_{0}^{1 / T} f^{*}(t) d t\right)^{2}+\int_{1 / T}^{\infty} f^{*}(t)^{2} d t,
$$

where the implied constant is absolute.

Note that the constant is even independent of $n$, the dimension. For $T \rightarrow \infty$ this reduces to Bessel's inequality. An analogous result for Fourier series is due to H. L. Montgomery, cf. our $§ 9$.

Proof. We only use the trivial estimate

$$
|\hat{f}(x)| \leqslant \int_{\mathbf{R}^{n}}|f(y)| d y=\int_{0}^{\infty} f^{*}(t) d t
$$

and Bessel's inequality

$$
\int_{\mathbf{R}^{n}}|\hat{f}(x)|^{2} d x \leqslant \int_{\mathbf{R}^{n}}|f(y)|^{2} d y=\int_{0}^{\infty} f^{*}(t)^{2} d t .
$$

Fix $s>0$ and decompose $f=f_{1}+f_{2}$ so that

$$
f_{1}^{*}(t)= \begin{cases}f^{*}(t) & \text { for } 0<t \leqslant s \\ 0 & \text { for } t>s\end{cases}
$$

and

$$
f_{2}^{*}(t)=f^{*}(t+s) \text { for } t>0
$$

This is the usual procedure in Marcinkiewicz type interpolation: If $f^{*}(s)=0$ we take $f_{1}=f$; if $f^{*}(s)>0$ we take $f_{1}=f$ on the $y$-set where $|f(y)|>f^{*}(s)$ and on a part of the $y$-set where $|f(y)|=f^{*}(s)$, but $f_{1}=0$ elsewhere. The second $y$-set is needed only if $f^{*}$ has an interval of constancy about $s$ and can be so selected that 
always $f_{1}=f$ on a set of measure $s$ (if we only count the nonzero values). Then $f_{1}$ and $f_{2}=f-f_{1}$ have the desired properties.

Now, for any measurable set $E$ of measure $|E|=T$ with characteristic function $\chi_{E}$ : we have

$$
\begin{aligned}
\int_{\mathbf{R}^{\prime \prime}}|\hat{f}|^{2} \chi_{E} & \ll \int_{\mathbf{R}^{n}}\left|\hat{f}_{1}\right|^{2} \chi_{E}+\int_{\mathbf{R}^{n}}\left|\hat{f}_{2}\right|^{2} \chi_{E} \ll T \sup \left|\hat{f}_{1}\right|^{2}+\int_{\mathbf{R}^{\prime \prime}}\left|\hat{f}_{2}\right|^{2} \\
& \ll T\left(\int_{0}^{\infty} f_{1}^{*}\right)^{2}+\int_{0}^{\infty} f_{2}^{* 2} \ll T\left(\int_{0}^{s} f^{*}\right)^{2}+\int_{s}^{\infty} f^{* 2}
\end{aligned}
$$

with absoulte constants (which can easily be given). Since

$$
\int_{0}^{T} \hat{f}^{*}(t)^{2} d t=\sup _{|E|=T} \int_{\mathbf{R}^{n}}|\hat{f}|^{2} \chi_{E}
$$

the result follows by choosing $s=1 / T$.

Next we formulate (3.1) so that it becomes more convenient for our applications. Denote the right side of $(3.1)$ by $B(T)$ for the moment. Since

$$
\int_{0}^{1 / t} f^{*} \geqslant \int_{0}^{1 / T} f^{*} \text { for } 0<t \leqslant T,
$$

we get

$$
B(T) \leqslant \int_{0}^{T}\left[\int_{0}^{1 / t} f^{*}\right]^{2} d t+\int_{0}^{T}\left[f^{*}\left(\frac{1}{t}\right)\right]^{2} \frac{d t}{t^{2}}
$$

but also $\int_{0}^{1 / t} f^{*} \geqslant(1 / t) f^{*}(1 / t)$ for $t>0$ and so we have with an absolute constant

$$
B(t) \ll \int_{0}^{T}\left(\int_{0}^{1 / t} f^{*}\right)^{2} d t
$$

With a little more work we can show

$$
B(T) \asymp \int_{0}^{T}\left(\int_{0}^{1 / t} f^{*}\right)^{2} d t
$$

but this follows later anyway. On the other hand, by Hardy's inequality, we see that

$$
\int_{0}^{T} \hat{f}^{(*)}(t)^{2} d t \ll \int_{0}^{T} \hat{f}^{*}(t)^{2} d t
$$

with an absolute constant; in fact, we have $\asymp$ since $\hat{f}^{(*)} \geqslant \hat{f}^{*}$. Thus we obtain

Corollary 1. For $T>0, f \in L^{2}\left(\mathbf{R}^{n}\right)$ we have

$$
\begin{aligned}
& \int_{0}^{T} \hat{f}^{(*)}(t)^{2} d t \ll \int_{0}^{T}\left(\int_{0}^{1 / t} f^{*}\right)^{2} d t=\int_{1 / T}^{\infty} f^{(*)}(t)^{2} d t, \\
& \int_{T}^{\infty} \hat{f}^{(*)}(t)^{2} d t \gg \int_{0}^{1 / T} f^{(*)}(t)^{2} d t=\int_{T}^{\infty}\left(\int_{0}^{1 / t} f^{*}\right)^{2} d t
\end{aligned}
$$

with absolute constants.

Inequality (3.6) follows from (3.5) by replacing $f$ and $T$ by $\hat{f}$ and $1 / T$, respectively. The introduction of the averaged rearrangements $\hat{f}^{(*)}$ and $f^{(*)}$ has brought about this perfect symmetry of the estimates. 
4. The basic local lower estimate. We prove the following lower estimate for positive functions.

THEOREM 2. For $T>0,0 \leqslant f \in L^{1}\left(\mathbf{R}^{n}\right)$ we have

$$
\int_{|N| \leqslant T}|\hat{f}(x)| d x \gg T^{n} \int_{|,| \leqslant n / T} f(y+z) d y \quad\left(z \in \mathbf{R}^{n}\right)
$$

with a constant depending only on $n$.

Proof. We consider Fejér's kernel

$$
K(y)=\prod_{j=1}^{n}\left(\frac{\sin \pi y_{j}}{\pi y_{j}}\right)^{2}=\hat{k}(y), \quad k(x)=\prod_{j=1}^{n}\left(1-\left|x_{j}\right|\right)^{+} .
$$

Since $T^{\prime \prime} K(T y)$ is the Fourier transform of $k(x / T)$ we obtain, by Fubini's theorem,

$$
\int_{\mathbf{R}^{n}} k\left(\frac{x}{T}\right) e(-x \cdot z) \hat{f}(x) d x=T^{n} \int_{\mathbf{R}^{\prime \prime}} K(T y) f(y+z) d y .
$$

It is convenient to use the square norm $|x|_{\infty}=\max \left|x_{j}\right|$ besides the euclidean norm $|x|=|x|_{2}$. Observe that $K \geqslant 0$ and

$$
K(y) \geqslant c^{n} \text { for }|y|_{\infty} \leqslant 1 / 2 .
$$

Hence (4.2) implies

$$
\int_{|x|_{\infty} \leqslant T}|\hat{f}(x)| d x \geqslant c^{n} \sup _{z} T^{n} \int_{\mid y_{\infty} \leqslant 1 / 2 T} f(y+z) d y .
$$

Inside the sup occur averages of $f$ over every cube with side $1 / T$, and such a sup becomes only smaller if $1 / T$ is replaced by $m / T, m \in \mathbf{N}$. Since the integral is monotone in the range $m / 2 T \leqslant 1 / T_{1} \leqslant(m+1) / 2 T$, we get for any $T_{1}, 0<T_{1} \leqslant$ $2 T$,

$$
\int_{|x|_{\infty} \leqslant T}|\hat{f}(x)| d x \geqslant c^{n} T_{1}^{n} \int_{\mid y_{\infty} \leqslant 1 / T_{1}} f(y+z) d y
$$

with another $c$. The result follows by applying (4.3) with $T$ and $T_{1}$ replaced by $T / \sqrt{n}$ and $T / n$.

REMARK. The proof indicates that the square metric is more natural in this context and leads to better constants, cf. (4.3).

For an application of (4.1) we need two corollaries.

CoRollary 2. If $0 \leqslant f \in L^{1}\left(\mathbf{R}^{n}\right)$ then for $x \neq 0$

$$
\mathbf{A} \hat{f}^{[*]}(x) \geqslant \mathbf{A}|\hat{f}|(x) \gg g(x)=\int_{|y| \leqslant \gamma_{n}^{-2 / n}|x|^{-1}} f(y) d y
$$

with a constant depending only on $n$.

This follows by Theorem 2 since $\gamma_{n} \geqslant(1 / \sqrt{n})^{n}$, hence $\gamma_{n}^{-2 / n} \leqslant n$. Note that $g$ is radial, $g(x)=G\left(\gamma_{n}|x|^{n}\right)$, with

$$
G(t)=\int_{|y| \leqslant\left(\gamma_{n} t\right)^{-1 / n}} f(y) d y \quad(t>0) .
$$


Corollary $2^{\prime}$. If $f \in L^{1}\left(\mathbf{R}^{n}\right)$ and $\phi=f^{[*]}$ then

$$
\hat{\phi}^{(*)}(t) \gg \int_{0}^{1 / t} f^{*}(s) d s \quad(t>0)
$$

with a constant depending only on $n$.

This follows if we apply (4.4) to $\phi$ since, in that case,

$$
\mathbf{A} \hat{\phi}^{[*]}(x)=\hat{\phi}^{(*)}\left(\gamma_{n} s^{n}\right), \quad g(x)=\int_{0}^{\left(\gamma_{n} s^{n}\right)^{-1}} f^{*} \quad(s=|x|) .
$$

Remarks. The constant in (4.5) can be improved if we replace $\phi=f^{|*|}$, the decreasing rearrangement over "circles", by $\phi=f^{\text {‘*}}$ ', the decreasing rearrangement over "squares", which is defined analogously using $|x|_{x}$ instead of $|x|_{2}$. A similar remark holds for (4.4).

Application. From (4.5) we see that (3.5) is maximal because, for $\phi=f^{|*|}$, the left side reaches the right side (at least for test functions). Since $B(T)$ (the right side of (3.1)) lies inbetween, (3.4) also follows. So, in addition, (3.1) is maximal. Again, it seems that $\phi=f^{6 *}$, would be an even better choice to show this.

5. A convexity principle. There is a general principle that permits us to derive, from (3.1) or (3.5) for fixed $f$ and all $T>0$, many further inequalities for $f$.

THEOREM 3. Assume that $\Psi(s) \geqslant 0$ is increasing and convex for $s \geqslant 0$, and similarly that $\Phi(s) \geqslant 0$ is increasing and concave, further let $U(t), G(t), H(t)$ be nonnegative and measurable for $t>0$. If $G$ decreases, then

$$
\int_{0}^{T} G(t) U(t) d t \leqslant \int_{0}^{T} H(t) U(t) d t<\infty \quad \text { for all } T>0
$$

implies

$$
\int_{0}^{T} \Psi(G(t)) U(t) d t \leqslant \int_{0}^{T} \Psi(H(t)) U(t) d t \quad \text { for all } T>0 .
$$

If $H$ increases, then (5.1) implies

$$
\int_{0}^{T} \Phi(G(t)) U(t) d t \leqslant \int_{0}^{T} \Phi(H(t)) U(t) d t \quad \text { for all } T>0 .
$$

[The integrals in (5.2) and (5.3) are permitted to be $+\infty$ ].

There are similar results for integrals $\int_{T}^{\infty}$ which follow by substitution. In the case of $U(t)=1$, the analogue of (5.2) for sums is due to R. M: Gabriel [2, Lemma 2]. In that case one can obtain (5.2) also from the necessary and sufficient conditions of $A$. Baernstein [1, Proposition 3], which are variants of similar results of Hardy, Littlewood and Polyá [5]. This procedure was used by H. L. Montgomery [9] without stating the above result explicitly. A similar approach is possible for (5.3). It is simpler, however, to give a direct proof: since $\Psi(s)=\Psi(0)+\int_{0}^{s} \Psi^{\prime}(t) d t$ we can obtain (by approximating $\Psi^{\prime}$ from below) a sequence $\Psi_{n}(s) \nearrow \Psi(s)$ for $s \geqslant 0$ satisfying the same assumptions as $\Psi$ and being continuously differentiable. So we may assume, without loss of generality, that $\Psi^{\prime}$ is continuous ( $\geqslant 0$ and increasing) and similarly that $\Phi^{\prime}$ is continuous ( $\geqslant 0$ and decreasing). Furthermore, by approximating $G$ from below, we may assume in the first part that $G(t)$ remains 
bounded as $t \rightarrow+0$; we may also normalize $G(t)$ so that it becomes left-continuous for $t>0$. Similarly, we may normalize $H(t)$ in the second part so that it becomes left-continuous for $t>0$.

Now observe that, for any pair $a \geqslant 0, b \geqslant 0$,

$$
\Psi(b)-\Psi(a) \geqslant \Psi^{\prime}(a)(b-a), \quad \Phi(b)-\Phi(a) \geqslant \Phi^{\prime}(b)(b-a) .
$$

Hence in the first part, for $t>0$,

$$
\Psi(H(t)) \geqslant \Psi(G(t))+\psi(t)(H(t)-G(t)), \quad 0 \leqslant \psi(t)=\Psi^{\prime}(G(t)) \searrow,
$$

and in the second part, for $t>0$,

$$
\Phi(H(t)) \geqslant \Phi(G(t))+\phi(t)(H(t)-G(t)), \quad 0 \leqslant \phi(t)=\Phi^{\prime}(H(t)) \searrow .
$$

Furthermore, $\psi(t)$ and $\phi(t)$ are left-continuous and bounded, since in the second part $H(t)$ remains clearly bounded as $t \rightarrow+0$. So the functions $\psi(H-G) U$ and $\phi(H-G) U$ are locally integrable and satisfy

$$
\int_{0}^{T} \psi(H-G) U \geqslant 0, \quad \int_{0}^{T} \phi(H-G) U \geqslant 0 \text { for } T>0
$$

in view of (5.1). But this implies (5.2) and (5.3).

We also need an $n$-dimensional version of Theorem 3 .

THEOREM 3 '. Assume that $\Psi(s) \geqslant 0$ is increasing and convex for $s \geqslant 0$, and similarly that $\Phi(s) \geqslant 0$ is increasing and concave, further let $u(x), g(x)$ and $h(x)$ be nonnegative and measurable for $x \in \mathbf{R}^{n}$. If $g$ is radial with decreasing $G$ then

$$
\int_{|x| \leqslant T} g(x) u(x) d x \leqslant \int_{|x| \leqslant T} h(x) u(x) d x<\infty \quad \text { for all } T>0
$$

implies

$$
\int_{|x| \leqslant T} \Psi(g(x)) u(x) d x \leqslant \int_{|x| \leqslant T} \Psi(h(x)) u(x) d x \quad \text { for all } T>0 .
$$

If $h$ is radial with increasing $H$ then (5.5) implies

$$
\int_{|x| \leqslant T} \Phi(g(x)) u(x) d x \leqslant \int_{|x| \leqslant T} \Phi(h(x)) u(x) d x \quad \text { for all } T>0 .
$$

[The integrals in (5.6) and (5.7) are permitted to be $+\infty$ ].

Proof. We can make the same additional assumptions about $\Psi, \Phi, G, H$ as we did in the proof of Theorem 3. Observe that $\psi(x)=\Psi^{\prime}(g(x)), \phi(x)=\Phi^{\prime}(h(x))$ are nonnegative bounded radial functions and that their associated functions are decreasing and left-continuous. So, instead of (5.4), we obtain

$$
\int_{|x| \leqslant T} \psi \cdot(h-g) u \geqslant 0, \quad \int_{|x| \leqslant T} \phi \cdot(h-g) u \geqslant 0 \text { for } T>0,
$$

since $\psi$ and $\phi$ can be approximated from below by linear combinations (with positive coefficients) of characteristic functions of closed balls. Now the result follows in the same way. 
6. General weighted upper estimates. The basic local upper estimates combined with the convexity principle leads to inequalities which seem to contain all previously known results which were usually obtained by interpolation.

THEOREM 4. Assume that $\Psi(s) \geqslant 0$ is an increasing convex function of $s^{2}$ for $s \geqslant 0$, and similarly that $\Phi(s) \geqslant 0$ is an increasing concave function of $s^{2}$; further let $f \in L^{2}\left(\mathbf{R}^{n}\right)$, and $W$ and $V$ be nonnegative and related by $V(t)=t^{-2} W(1 / t)$ for $t>0$. Then we have, in case that $W \searrow$,

$$
\begin{gathered}
\int_{0}^{\infty} \Psi\left(\hat{f}^{(*)}\right) W \leqslant \int_{0}^{\infty} \Psi\left(C \int_{0}^{1 / t} f^{*}\right) W=\int_{0}^{\infty} \Psi\left(C t f^{(*)}(t)\right) V, \\
\int_{0}^{\infty} \Psi\left(t \hat{f}^{(*)}(t)\right) V \geqslant \int_{0}^{\infty} \Psi\left(c f^{(*)}\right) W=\int_{0}^{\infty} \Psi\left(c t \int_{0}^{1 / t} f^{*}\right) V,
\end{gathered}
$$

and in case that $V \nearrow$,

$$
\begin{gathered}
\int_{0}^{\infty} \Phi\left(t \hat{f}^{(*)}(t)\right) W \leqslant \int_{0}^{\infty} \Phi\left(C t \int_{0}^{1 / t} f^{*}\right) W=\int_{0}^{\infty} \Phi\left(C f^{(*)}\right) V, \\
\int_{0}^{\infty} \Phi\left(\hat{f}^{(*)}\right) V \geqslant \int_{0}^{\infty} \Phi\left(c t f^{(*)}(t)\right) W=\int_{0}^{\infty} \Phi\left(c \int_{0}^{1 / t} f^{*}\right) V
\end{gathered}
$$

with absolute constants $C, c$.

Proof. By approximation from below we may assume $W$ to be bounded in the first part, and $V$ to be bounded in the second part. To derive (6.1) let $U(t)=W(t)$, $G(t)=\hat{f}^{*}(t)^{2}$, and $H(t)=\left(c \int_{0}^{1 / t} f^{*}\right)^{2}$. From (3.5) we see that

$$
\int_{0}^{T} G \leqslant \int_{0}^{T} H<\infty, \text { hence } \int_{0}^{T} G U \leqslant \int_{0}^{T} H U<\infty \text { for } T>0 \text {. }
$$

Now Theorem 3 yields the result $(T \rightarrow+\infty)$.

To derive (6.3) let $U(t)=W(t), G(t)=\left[t \hat{f}^{(*)}(t)\right]^{2}$, and $H(t)=\left(C t \int_{0}^{1 / t} f^{*}\right)^{2}$ which is increasing. From (3.5) we see that

$$
\int_{0}^{T} G \frac{d t}{t^{2}} \leqslant \int_{0}^{T} H \frac{d t}{t^{2}}<\infty
$$

hence

$$
\int_{0}^{T} G V\left(\frac{1}{t}\right) \frac{d t}{t^{2}} \leqslant \int_{0}^{T} H V\left(\frac{1}{t}\right) \frac{d t}{t^{2}}<\infty \quad(T>0) .
$$

Again Theorem 3 yields the result since $t^{-2} V(1 / t)=W(t)=U(t)$.

If we replace $f$ by $\hat{f}$ then (6.1) turns into (6.2), and (6.3) turns into (6.4). So the proof is complete.

REMARKS. If we take, for $W$, the characteristic function of $(0, T]$ or, for $V$, the characteristic function of $[1 / T, \infty)$, we obtain the following special cases of $(6.1)$ to (6.4):

$$
\begin{gathered}
\int_{0}^{T} \Psi\left(\hat{f}^{(*)}\right) \leqslant \int_{0}^{T} \Psi\left(C \int_{0}^{1 / t} f^{*}\right)=\int_{1 / T}^{\infty} \Psi\left(C t f^{(*)}(t)\right) \frac{d t}{t^{2}} \\
\int_{T}^{\infty} \Psi\left(t \hat{f}^{(*)}(t)\right) \frac{d t}{t^{2}} \geqslant \int_{0}^{1 / T} \Psi\left(c f^{(*)}\right)=\int_{T}^{\infty} \Psi\left(c t \int_{0}^{1 / t} f^{*}\right) \frac{d t}{t^{2}}
\end{gathered}
$$




$$
\begin{gathered}
\int_{0}^{T} \Phi\left(t \hat{f}^{(*)}(t)\right) \frac{d t}{t^{2}} \leqslant \int_{0}^{T} \Phi\left(C t \int_{0}^{1 / t} f^{*}\right) \frac{d t}{t^{2}}=\int_{1 / T}^{\infty} \Phi\left(C f^{(*)}\right), \\
\int_{T}^{\infty} \Phi\left(\hat{f}^{(*)}\right) \geqslant \int_{0}^{1 / T} \Phi\left(c t f^{(*)}(t)\right) \frac{d t}{t^{2}}=\int_{T}^{\infty} \Phi\left(c \int_{0}^{1 / t} f^{*}\right),
\end{gathered}
$$

each for all $T>0$. This is the "local" form of our inequalities. If we integrate them with respect to a positive measure we obtain the corresponding "weighted" from as in Theorem 4. So both forms are equivalent.

The analogue of (6.5) for Fourier series is due to H. L. Montgomery, cf. our $\S 9$. The other inequalities are new. The upper inequalities are maximal in the following sense: If we take $\phi=f^{[*]}, f \in L^{1}\left(\mathbf{R}^{n}\right)$, we infer, from Corollary $2^{\prime}$,

$$
\begin{gathered}
\int_{0}^{\infty} \Psi\left(\hat{\phi}^{(*)}\right) W \geqslant \int_{0}^{\infty} \Psi\left(c_{n} \int_{0}^{1 / t} f^{*}\right) W, \\
\int_{0}^{\infty} \Phi\left(t \hat{\phi}^{(*)}(t)\right) W \geqslant \int_{0}^{\infty} \Phi\left(c_{n} t \int_{0}^{1 / t} f^{*}\right) W .
\end{gathered}
$$

So if we vary $f$ in (6.1) or (6.3) without changing $f^{*}$ then the left sides reach the right sides for $f=\phi$ in the way described. Also (6.5) and (6.7) are maximal as special cases.

In connection with (6.1) we mention the following rearrangement inequalities for nonnegative $w$ with $w^{*}=W$

$$
\int_{\mathbf{R}^{n}} \Psi(|\hat{f}|) w \leqslant \int_{\mathbf{R}^{n}} \Psi\left(\hat{f}^{[*]}\right) w^{[*]}=\int_{0}^{\infty} \Psi\left(\hat{f}^{*}\right) W \leqslant \int_{\mathbf{R}^{n}} \Psi\left(\mathbf{A} \hat{f}^{[*]}\right) w^{[*]}=\int_{0}^{\infty} \Psi\left(\hat{f}^{(*)}\right) W .
$$

So all these terms are estimated in (6.1). Since

$$
\int_{0}^{T} \hat{f}^{*}(t)^{2} d t \leqslant \int_{0}^{T} \hat{f}^{(*)}(t)^{2} d t \leqslant C \int_{0}^{T} \hat{f}^{*}(t)^{2} d t
$$

it follows from Theorem 3 (same $\Psi$ and $W$ as before) that

$$
\int_{0}^{T} \Psi\left(\hat{f}^{*}\right) W \leqslant \int_{0}^{\infty} \Psi\left(\hat{f}^{(*)}\right) W \leqslant \int_{0}^{\infty} \Psi\left(C \hat{f}^{*}\right) W .
$$

So we can replace $\hat{f}^{(*)}$ in $(6.1)$ by $\hat{f}^{*}$ without any loss. This may not be the case in (6.3). But at least we have the following rearrangement inequalities for radial $w(x)=W\left(\gamma_{n}|x|^{n}\right)$ :

$$
\begin{gathered}
\int_{|y| \leqslant|x|}|\hat{f}(y)| d y \leqslant \int_{|y| \leqslant|x|} \hat{f}^{[*]}(y) d y=\int_{0}^{\gamma_{n}|x|^{n}} \hat{f}^{*}(t) d t, \\
\int_{\mathbf{R}^{n}} \Phi\left(\int_{|y| \leqslant|x|}|\hat{f}(y)| d y\right) w(x) d x \leqslant \int_{0}^{\infty} \Phi\left(\int_{0}^{t} \hat{f}^{*}\right) W(t) d t .
\end{gathered}
$$

So also, terms like $\int \Phi\left(|x|^{n} \mathbf{A}|\hat{f}|\right) w(x) d x$ can be estimated by (6.3). Repeating the proof, one further sees that terms like $\int \Phi\left(|x|^{n}|\hat{f}|\right) w(x) d x$ can again be estimated by the right side of (6.3), since this is so for $\Phi(s)=s^{2}$; but such estimates may not be maximal.

Next we discuss the special cases $\Psi(s)^{q}=s(2 \leqslant q<\infty), \Phi(s)=s^{p}(1<p \leqslant 2)$. We get, from Theorem 4 by combining the upper estimates and the lower estimates, 
Corollary 3. Let $1<q<\infty$ and suppose that

$$
0 \leqslant W(t) \searrow, \quad V(t)=t^{1-(2 / q)} W\left(\frac{1}{t}\right) \nearrow \quad(t>0) .
$$

Then there are absolute constants $C$ and $c$ such that

$$
\begin{gathered}
\int_{0}^{\infty}\left(W \hat{f}^{(*)}\right)^{q} \leqslant C^{q} \int_{0}^{\infty}\left(V f^{(*)}\right)^{q}, \\
\int_{0}^{\infty}\left(V \hat{f}^{(*)}\right)^{q} \geqslant c^{q} \int_{0}^{\infty}\left(W f^{(*)}\right)^{q}
\end{gathered}
$$

hold for $f \in L^{2}\left(\mathbf{R}^{n}\right)$.

These inequalities are "weighted" extensions of the usual inequalities of Hardy, Littlewood and Paley. The corresponding "local" forms (6.5), (6.7) look as follows $\left(T>0, f \in L^{2}\right)$ :

$$
\begin{array}{ll}
\int_{0}^{T} \hat{f}^{(*)}(t)^{q} d t \leqslant C^{q} \int_{1 / T}^{\infty} t^{q-2} f^{(*)}(t)^{q} d t & (2 \leqslant q<\infty), \\
\int_{0}^{T} t^{p-2} \hat{f}^{(*)}(t)^{p} d t \leqslant C^{p} \int_{1 / T}^{\infty} f^{(*)}(t)^{p} d t & (1<p \leqslant 2),
\end{array}
$$

and similarly for the lower inequalities. Note that for $p \rightarrow 1^{+}$both sides of $(6.14)$ go to $+\infty$ unless $f \equiv 0$. For $T \rightarrow \infty$ these inequalities turn into the usual inequalities of Hardy, Littlewood and Paley with the difference that the averaged rearrangements occur. This makes no difference on the left side of (6.13), but on the right side it permits the absolute constant $C$. This is lost if one replaces $f^{(*)}$ by $f^{*}$, as is well known.

Note that (3.5) is the case $q=2$ of (6.13), and just as (3.5) is equivalent to (3.1) there is the following equivalent form of $(6.13)$

$$
\int_{0}^{T} \hat{f}^{*}(t)^{q} d t \ll T\left(\int_{0}^{1 / T} f^{*}(t) d t\right)^{q}+\int_{1 / T}^{\infty} t^{q-2} f^{*}(t)^{q} d t
$$

with a constant depending only on $q$, but not necessarily of the form $C^{q}$. Likewise, (6.14) is equivalent to

$$
\int_{0}^{T} t^{p-2} \hat{f}^{*}(t)^{p} d t \ll T^{p-1}\left(\int_{0}^{1 / T} f^{*}(t) d t\right)^{p}+\int_{1 / T}^{\infty} f^{*}(t)^{p} d t
$$

with a constant depending only on $p$. It is interesting to observe that these two inequalities can also be proved directly from the special case $T=\infty$ by the interpolation procedure of Theorem 1. However, one obtains a better constant going via Theorem 3. This phenomenon was observed by H. L. Montgomery in the case of Fourier series, cf. our $\$ 9$.

Finally we note that instead of the maximal inequality (6.1) we can prove, e.g.,

$$
\begin{gathered}
\int_{0}^{\infty} \Psi\left(W \hat{f}^{*}\right) \leqslant \int_{0}^{\infty} \Psi\left(C W \int_{0}^{1 / t} f^{*}\right) d t \\
\int_{0}^{\infty} \Psi(W|\hat{\phi}|) \geqslant \int_{0}^{\infty} \Psi\left(c_{n} W \int_{0}^{1 / t} f^{*}\right) d t, \quad \phi=f^{[*]},
\end{gathered}
$$


for the same $\Psi$ and $W$, using (3.1) and Theorem 3 for (6.17) and (4.5), Hardy's inequality and Theorem 3 for (6.18). This is a natural extension of the maximal inequality (6.11).

7. General weighted lower estimates. The basic local lower estimate combined with the convexity principle leads to general lower inequalities which can, e.g., be used to show that certain weaker forms of (6.1) and (6.3) are still maximal.

THEOREM 5. Let $1<\alpha<\infty$ and assume that $\Psi(s) \geqslant 0$ is an increasing convex function at $s^{\alpha}$ for $s \geqslant 0$; further suppose that $w(x)=W\left(\gamma_{n}|x|^{n}\right), v(x)=V\left(\gamma_{n}|x|^{n}\right)$ satisfy

$$
0 \leqslant W(t) \searrow, \quad V(t)=t^{-2} W(1 / t) \text { for } t>0 .
$$

Then we have, for $0 \leqslant f \in L^{1}\left(\mathbf{R}^{n}\right)$,

$$
\int_{\mathbf{R}^{n}} \Psi\left(C_{\alpha}|\hat{f}|\right) w \geqslant \int_{\mathbf{R}^{n}} \Psi\left(c_{n}|x|^{n} \mathbf{A} f\right) v(x) d x
$$

with constants $C_{\alpha}$ and $c_{n}$ depending only on $\alpha$ and $n$, respectively.

Proof. By an $n$-dimensional version of Hardy's inequality we obtain

$$
\int_{|x| \leqslant T}(\mathbf{A}|\hat{f}|)^{\alpha} d x \leqslant \int_{|x| \leqslant T}\left(C_{\alpha}|\hat{f}|\right)^{\alpha} d x \quad(T>0)
$$

and in view of Corollary 2 (using the notation from there)

$$
\int_{|x| \leqslant T}\left(c_{n} g\right)^{\alpha} d x \leqslant \int_{|x| \leqslant T}\left(C_{\alpha}|\hat{f}|\right)^{\alpha} d x \quad(T>0) .
$$

Now Theorem $3^{\prime}$ applies and yields

$$
\int_{|x| \leqslant T} \Psi\left(c_{n} g\right) d x \leqslant \int_{|x| \leqslant T} \Psi\left(C_{\alpha}|\hat{f}|\right) d x
$$

or more generally

$$
\int_{\mathbf{R}^{n}} \Psi\left(c_{n} g\right) w \leqslant \int_{\mathbf{R}^{n}} \Psi\left(C_{\alpha}|\hat{f}|\right) w
$$

But this is the same as (7.1) with another $c_{n}$, since

$$
\begin{aligned}
\int_{\mathbf{R}^{n}} \Psi\left(c_{n} g\right) w & =\int_{0}^{\infty} \Psi\left(c_{n} G(t)\right) W(t) d t=\int_{0}^{\infty} \Psi\left(c_{n} G\left(\frac{1}{t}\right)\right) V(t) d t \\
& =\int_{\mathbf{R}^{n}} \Psi\left(c_{n} \gamma_{n}|x|^{n} \mathbf{A} f\right) v(x) d x .
\end{aligned}
$$

Remarks. Note that (7.2) is the local form of (7.1). In the special case that $f$ is radial, $f(x)=F\left(\gamma_{n}|x|^{n}\right)$, we simply have

$$
\int_{\mathbf{R}^{n}} \Psi\left(C_{\alpha}|\hat{f}|\right) w \geqslant \int_{0}^{\infty} \Psi\left(c_{n} \int_{0}^{t} F\right) V(t) d t=\int_{0}^{\infty} \Psi\left(c_{n} \int_{0}^{1 / t} F\right) W(t) d t
$$


Take $\alpha=2$ and compare this with

$$
\int_{\mathbf{R}^{n}} \Psi(|\hat{f}|) w \leqslant \int_{0}^{\infty} \Psi\left(C \int_{0}^{1 / t} f^{*}\right) W(t) d t
$$

a weakened form of (6.1). We see that (7.4) is still maximal by taking $f=f^{[*]}$. If we weaken (6.3) to

$$
\int_{\mathbf{R}^{\prime \prime}} \Phi\left(\int_{|y| \leqslant|x|}|\hat{f}(y)| d y\right) w(x) d x \leqslant \int_{0}^{\infty} \Phi\left(C t \int_{0}^{1 / t} f^{*}\right) W(t) d t,
$$

we can see, from Corollary 2 directly, that this is still maximal by taking $f=f^{[*]}$.

Of particular interest is the special case $\Psi(s)=s^{\alpha}$ with $\alpha=q$.

Corollary 4. Let $1<q<\infty$ and suppose that $w(x)=W\left(\gamma_{n}|x|^{n}\right), v(x)=$ $V\left(\gamma_{n}|x|^{n}\right)$ satisfy

$$
0 \leqslant W(t) \searrow, \quad V(t)=t^{1-(2 / q)} W\left(\frac{1}{t}\right) \text { for } t>0 .
$$

Then we have, for $0 \leqslant f \in L^{\mathrm{l}}\left(\mathbf{R}^{n}\right)$,

$$
\int_{\mathbf{R}^{n}}\left(C_{q} w|\hat{f}|\right)^{q} \geqslant \int_{\mathbf{R}^{n}}\left(c_{n} v \mathbf{A} f\right)^{q}
$$

with constants $C_{q}, c_{n}$ depending only on $q, n$, respectively.

This should be compared with the weakened form of (6.11), viz.

$$
\int_{0}^{\infty}\left(W \hat{f}^{*}\right)^{q} \leqslant C^{q} \int_{0}^{\infty}\left(V f^{(*)}\right)^{q}
$$

or, equivalently,

$$
\int_{\mathbf{R}^{n}}\left(w \hat{f}^{[*]}\right)^{q} \leqslant C^{q} \int_{\mathbf{R}^{n}}\left(v \mathbf{A} f^{[*]}\right)^{q}
$$

or, still weaker,

$$
\int_{\mathbf{R}^{n}}(w|\hat{f}|)^{q} \leqslant C^{q} \int_{\mathbf{R}^{n}}\left(v \mathbf{A} f^{[*]}\right)^{q} .
$$

It follows that these inequalities are still maximal by taking $f=f^{[*]}$. For $q \geqslant 2$ this follows also from (6.18).

8. Variants of the convexity principle. For the applications of Theorem 3 in $\S \S 6$ and 7 it would be natural to permit constants on the outside, i.e. to ask for inequalities of the type

$$
\int_{0}^{\infty} \Psi(G) U \ll \int_{0}^{\infty} \Psi(H) U, \quad \int_{0}^{\infty} \Phi(G) U \ll \int_{0}^{\infty} \Phi(H) U,
$$

where $\Psi(s)$ and $\Phi(s)$ are positive increasing and convex, concave, respectively, for $s>0$. So we should like to replace $\Psi(s)$ and $\Phi(s)$ by positive continuous functions $\psi(s)$ and $\phi(s)$ such that

$$
\psi(s) \asymp \Psi(s), \quad \phi(s) \preceq \Phi(s) \text { for } s>0 .
$$

[In the applications we also need the existence of $\psi(+0)$ and $\phi(+0)$.] 
In this section we discuss sufficient conditions for positive continuous functions $\psi$ and $\phi$ so that there exist positive increasing convex or concave functions $\Psi$ and $\Phi$ satisfying (8.1).

First we note that the condition (8.1) separates into a condition for small $s$ and into another independent condition for large $s$ : Let $0<a<b<\infty$ and suppose that $\Psi_{1}$ and $\Phi_{1}$ are positive increasing and convex, concave, resp., on $(0, a]$, while $\Psi_{2}$ and $\Phi_{2}$ are nonconstant positive increasing and convex, concave, resp., on $[b, \infty)$. Further assume that $\psi(s)$ and $\phi(s)$ are positive, continuous for $s>0$, satisfying

$$
\begin{array}{lll}
\psi(s) \asymp \Psi_{1}(s), & \phi(s) \asymp \Phi_{1}(s) & \text { on }(0, a], \\
\psi(s) \asymp \Psi_{2}(s), & \phi(s) \asymp \Phi_{2}(s) & \text { on }[b, \infty) .
\end{array}
$$

Then there exist positive increasing convex, resp. concave functions $\Psi(s)$ and $\Phi(s)$ for $s>0$ such that (8.1) holds. We can take, e.g.,

$$
\Psi(s)= \begin{cases}\psi_{1}(s) & \text { for } 0<s \leqslant a, \\ \psi_{1}(a)+m(s-a) & \text { for } a \leqslant s \leqslant b, \\ c \psi_{2}(s)+d & \text { for } s \geqslant b,\end{cases}
$$

where we select $m=\psi_{1}^{\prime}\left(a^{-}\right), c=m / \psi_{2}^{\prime}\left(b^{+}\right)$, and then $d$ so that the definitions at $s=b$ match. (Make $b$ large if necessary to ensure that $\psi_{2}^{\prime}\left(b^{+}\right)>0$.) Then the positive increasing convex function $\psi_{1}$ is first extended linearly by an increasing linear function and then extended by an increasing convex function coming from $\psi_{2}$. The same construction gives $\Phi$. It is easy to see that (8.1) holds.

Next we are concerned with simple sufficient conditions for (8.2).

Lemma 1. Suppose that the positive continuous function $\psi$ satisfies

$$
\begin{aligned}
& \psi(s) \asymp s^{\alpha_{1}} L_{1}(s) \quad \text { as } s \rightarrow+0, \\
& \psi(s) \asymp s^{\alpha_{2}} L_{2}(s) \quad \text { as } s \rightarrow+\infty,
\end{aligned}
$$

where $\alpha_{1}, \alpha_{2}>1$, and $L_{1}(s)$ and $L_{2}(s)$ are positive slowly varying functions. Then $\psi$ satisfies (8.2) with suitable $a, b$. A corresponding result holds for $\phi$ with exponents $0<\alpha_{1}<1,0<\alpha_{2}<1$.

Proof. Let $\theta_{1}(s)=s^{\alpha_{1}} L_{1}(s), \theta_{2}(s)=s^{\alpha_{2}} L_{2}(s)$. To construct $\Psi_{1}$ we define

$$
\psi_{1}\left(\frac{1}{2^{k}}\right)=\theta_{1}\left(\frac{1}{2^{k}}\right) \text { for } k \geqslant k_{0}
$$

and connect these values linearly. Observe that for $s \rightarrow+0$

$$
\begin{aligned}
\theta_{1}(2 s) & =\left(2^{\alpha_{1}}+o(1)\right) \theta_{1}(s), \\
\frac{\theta_{1}(2 s)-\theta_{1}(s)}{s} & =\left(2^{\alpha_{1}}-1+o(1)\right) s^{\alpha_{1}-1} L_{1}(s) \geqslant \frac{\theta_{1}(s)-\theta_{1}(s / 2)}{s / 2}
\end{aligned}
$$

since $L_{1}(2 s) / L_{1}(s) \rightarrow 1$. Therefore, $\Psi_{1}$ is positive increasing and convex if we take $k_{0}$ large enough. Moreover, $\Psi_{1}(s) \asymp \theta_{1}(s)$ as $s \rightarrow+0$. The construction of $\Phi_{1}$ is the same. For $\Psi_{2}$ and $\Phi_{2}$ we interpolate the values $\theta_{2}\left(2^{k}\right)$. 
In order to permit other exponents $\alpha_{1}, \alpha_{2}=1$ we turn to logarithmco-exponential functions $E$ in the sense of Hardy [3].

LEMMA 2. If the positive continuous function $\psi$ satisfies

$$
\begin{array}{ll}
\psi(s) \asymp E_{1}(s) \ll s & (s \rightarrow+0), \\
\psi(s) \asymp E_{2}(s) \gg s & (s \rightarrow+\infty),
\end{array}
$$

with logarithmco-exponential functions $E_{1}$ and $E_{2}$, then $\psi$ satisfies (8.2) with suitable $a, b$. If the positive continuous function $\phi$ satisfies

$$
\begin{array}{lll}
\phi(s) \asymp E_{1}(s), & s \ll E_{1}(s) \ll 1 & (s \rightarrow+0), \\
\phi(s) \asymp E_{2}(s), & 1 \ll E_{2}(s) \ll s & (s \rightarrow+\infty),
\end{array}
$$

then $\phi$ satisfies (8.2) with suitable $a, b$.

Proof. Using the fact that derivatives of logarithmco-exponential functions have eventually fixed sign, we see that $E_{1}$ in (8.4) is either positive increasing and convex itself or $E_{1} \asymp s$, in which case we replace $E_{1}$ by $s$. Then we may take $\psi_{1}=E_{1}$. Similarly, $E_{2}$ in (8.4) is either nonconstant positive increasing and convex or $E_{2} \asymp s$. Finally, $E_{1}$ and $E_{2}$ in (8.5) are either nonconstant positive increasing and concave or $\asymp s$ or $\measuredangle 1$. The choices of $\Phi_{1}$ and $\Phi_{2}$ are clear unless $E_{2} \asymp 1$, but then we take $\Phi_{2}(s)=1-1 / s(s \rightarrow+\infty)$.

REMARK. Note that the following conditions are necessary in the context of (8.2):

$$
\begin{array}{lll}
\Psi_{1}(s) \ll s & \text { as } s \rightarrow+0 & \left(\text { if } \Psi_{1}(0)=0\right), \\
s \ll \Phi_{1}(s) \ll 1 & \text { as } s \rightarrow+0 & \left(\text { if } \Phi_{1}(0)=0\right), \\
s \ll \Psi_{2}(s) & \text { as } s \rightarrow+\infty & \left(\text { since } \Psi_{2} \text { is nonconstant }\right), \\
1 \ll \Phi_{2}(s) \ll s & \text { as } s \rightarrow+\infty &
\end{array}
$$

So the extra conditions on $E_{1}$ and $E_{2}$ in Lemma 2 cannot be avoided. That means that our question (8.1) is completely answered for functions $\psi$ and $\phi$ which show logarithmco-exponential behavior at 0 and at $\infty$; and in the more general case of power behavior modified by slowly varying factors, we have simple sufficient conditions. This can be applied, in particular, to Theorems 4 and 5 and increases the range of these inequalities.

9. Analogues for Fourier series. We use a separate notation in this and the following section: Let $f \in L^{2}[0,1]$ have the Fourier expansion

$$
f(x) \sim \sum_{-\infty}^{\infty} a_{n} e(n x),
$$

and let $f^{*}$ denote the decreasing rearrangement of $|f|$ on $[0,1]$; similarly let $a_{n}^{*}$, $n \geqslant 0$, be the decreasing rearrangement of $\left|a_{n}\right|,-\infty<n<\infty$ (neglecting zeros if necessary). Then the basic local upper estimate of H. L. Montgomery [9] is

$$
\int_{0}^{T} f^{*}(t)^{2} d t \ll T\left(\sum_{n \leqslant 1 / T} a_{n}^{*}\right)^{2}+\sum_{n>1 / T} a_{n}^{* 2} \quad(0<T \leqslant 1),
$$


where the implied constant is absolute. Introduce further

$$
\begin{aligned}
a^{*}(s) & =a_{n}^{*} \quad \text { for } n \leqslant s<n+1, n \geqslant 0, \\
\beta(t) & =\int_{0}^{1 / t} a^{*}(s) d s, \quad t>0, \\
g(x) & =\sum_{n=0}^{\infty} a_{n}^{*} e(n x), \quad 0<x<1 .
\end{aligned}
$$

The right side of $(9.1)$ is (with absolute constants)

$$
\asymp \int_{0}^{T} \beta(t)^{2} d t \asymp \int_{0}^{T}|g(x)|^{2} d x \quad(0<T \leqslant 1)
$$

(note that $\int_{0}^{T} \beta(t)^{2} d t \ll \int_{0}^{T}|g(x)|^{2} d x$ follows by (9.4) and Hardy's inequality), and therefore (9.1) is maximal: the left side reaches the right side for $f=g$ (consider $a_{n}^{*}$ given and vary $a_{n}$ ). It is convenient to use Hardy's inequality to reformulate (9.1) as follows:

$$
\int_{0}^{T} f^{(*)}(t)^{2} d t \ll \int_{0}^{T} \beta(t)^{2} d t=\int_{1 / T}^{\infty} a^{(*)}(s)^{2} d s \quad(0<T \leqslant 1),
$$

where

$$
\begin{array}{ll}
f^{(*)}(t)=\frac{1}{t} \int_{0}^{t} f^{*}(x) d x, & 0<t \leqslant 1, \\
a^{(*)}(t)=\frac{1}{t} \int_{0}^{t} a^{*}(s) d s, & t>0 .
\end{array}
$$

Analogously to Theorem 2 we can prove the following basic local lower estimate in case that all $a_{n} \geqslant 0$ :

$$
\int_{0}^{T}|f(x)| d x \gg T \sum_{|n| \leqslant 2 / T} a_{n} \quad(0<T \leqslant 1)
$$

where the implied constant is absolute ( $f \in L^{2}$ or $f \in L^{1}$ ). For decreasing $a_{n}$ this can also be obtained by following Hardy and Littlewood [4, p. 7]; cf. also Zygmund [12, II, p. 129]. It is convenient to use

$$
a(s)=a_{[s]}, \quad-\infty<s<\infty,
$$

in order to write the right side of (9.3) as an integral. In the special case $f=g$ we have with an absolute constant

$$
g^{(*)}(T) \geqslant \frac{1}{T} \int_{0}^{T}|g(x)| d x \gg \int_{0}^{1 / T} a^{*}(s) d s=\beta(T) \quad(0<T \leqslant 1) .
$$

Since Theorem 3 is also valid for finite intervals we now obtain the following analogue of Theorems 4 and 5 . Here we use the additional notation

$$
\begin{aligned}
b(t) & =\int_{-1 / t}^{1 / t} a(s) d s, & t>0, \\
\mathbf{A} a(t) & =\frac{1}{2 t} \int_{-t}^{t} a(s) d s, & t>0 .
\end{aligned}
$$


THEOREM 6. Assume that $f \in L^{2}[0,1]$ and $V(t)=t^{-2} W(1 / t)$ for $t \geqslant 1$. Now we have three cases:

(i) If $0 \leqslant W(t) \searrow(0<t \leqslant 1)$ and $\Psi(s) \geqslant 0$ is an increasing convex function of $s^{2}$ for $s \geqslant 0$, then

$$
\int_{0}^{1} \Psi\left(f^{(*)}\right) W \leqslant \int_{0}^{1} \Psi(C \beta) W=\int_{1}^{\infty} \Psi\left(C t a^{(*)}(t)\right) V .
$$

(ii) If $0 \leqslant V(t) \nearrow(t \geqslant 1)$ and $\Phi(s) \geqslant 0$ is an increasing concave function of $s^{2}$ for $s \geqslant 0$, then

$$
\int_{0}^{1} \Phi\left(t f^{(*)}(t)\right) W \leqslant \int_{0}^{1} \Phi(c t \beta(t)) W=\int_{1}^{\infty} \Phi\left(C a^{(*)}\right) V .
$$

(iii) If $0 \leqslant W(t) \searrow(0<t \leqslant 1), 1<\alpha<\infty$, and $\Psi(s) \geqslant 0$ is an increasing convex function of $s^{\alpha}$ for $s \geqslant 0$, then

$$
\int_{0}^{1} \Psi(|f|) W \geqslant \int_{0}^{1} \Psi\left(c_{\alpha} b\right) W=\int_{1}^{\infty} \Psi\left(2 c_{\alpha} t \mathbf{A} a\right) V,
$$

provided that all $a_{n} \geqslant 0$.

[Here $C$ is an absolute constant and $c_{\alpha}$ depends only in $\alpha$. ]

The proofs of (9.5), (9.6) and (9.7) parallel those of (6.1), (6.3) and (7.1), respectively. There are equivalent local forms of these inequalities. The local form of (9.5) is due to H. L. Montgomery [9]; the other inequalities are new. The inequalities (9.5) and (9.6) are maximal in view of (9.4). In (9.5) one can replace $f^{(*)}$ by $f^{*}$ without loss, but even the weaker inequality with $|f|$ in place of $f^{(*)}$ is maximal in view of (9.7). In (9.6) one can also replace $f^{(*)}$ by $f^{*}$ or $|f|$, but these inequalities may not be maximal anymore. However, if we replace $t f^{(*)}(t)=\int_{0}^{t} f^{*}$ by $\int_{0}^{t}|f|$ the inequality remains maximal.

Particularly interesting are the special cases $\Psi(s)=s^{q}(2 \leqslant q<\infty), \Phi(s)=s^{p}$ $(1<p \leqslant 2)$, and $\Psi(s)=s^{\alpha}$ of (9.5), (9.6) and (9.7), respectively. We only mention the first two local forms using the notation

$$
\begin{aligned}
& a_{n}^{(*)}=\frac{1}{n+1} \sum_{k=0}^{n} a_{k}^{*}, \quad n \geqslant 0 ; \\
& \int_{0}^{T} f^{(*)}(t)^{q} d t \leqslant C^{q} \sum_{n \geqslant 1 / T} n^{q-2} a_{n}^{(*) q} \quad(0<T \leqslant 1), \\
& \int_{0}^{T} t^{p-2} f^{(*)}(t)^{p} d t \leqslant C^{p} \sum_{n \geqslant 1 / T} a_{n}^{(*) p} \quad(0<T \leqslant 1) .
\end{aligned}
$$

These are local versions of the inequalities of Hardy, Littlewood and Paley. The averaged rearrangement on the right permits the occurrence of the absolute constant. Inequality (9.8) for $T=1$ is due to Littlewood [7], who observed this phenomenon first. The averaged rearrangements on the left are less essential. If $p \rightarrow 1^{+}$both sides of (9.9) becomes infinite unless $f \equiv 0$. Finally we mention that (9.1) can be extended to orthogonal series, and the same is true for the more general upper estimates derived from it, in particular for (9.8) and (9.9). 
10. Analogues for Fourier coefficients. We briefly discuss Fourier coefficients (the inverse operation to forming a Fourier series) since this is not automatically implied as in the case of Fourier transforms. Here the basic local upper estimate is

$$
\sum_{n \leqslant N} a_{n}^{* 2} \ll N\left(\int_{0}^{1 / N} f^{*}(t) d t\right)^{2}+\int_{1 / N}^{1} f^{*}(t)^{2} d t \quad(N \text { real } \geqslant 1)
$$

for all $f \in L^{2}[0,1]$, the implied constant being absolute. The proof parallels that of Theorem 1 and is based only on Bessel's inequality and the trivial estimate. Therefore (10.1) can be extended to orthogonal series. We can rewrite (10.1) as follows (using Hardy's inequality):

$$
\int_{1}^{N} a^{(*)}(s)^{2} d s \ll \int_{1}^{N}\left(\int_{0}^{1 / s} f^{*}\right)^{2} d s=\int_{1 / N}^{1} f^{(*)}(t)^{2} d t \quad(N \geqslant 1),
$$

the implied constant being absolute $\left(f \in L^{2}\right.$ ). In the range $N \in[1,2]$ this should be checked directly.

Analogously to Theorem 2 we can prove the following basic local lower estimate in case that $f \geqslant 0$ on $[0,1]$ :

$$
\sum_{|n| \leqslant N}\left|a_{n}\right| \gg N \int_{0}^{1 / N} f(x) d x \quad(N \text { real } \geqslant 1)
$$

the implied constant being absolute ( $f \in L^{1}$ or $L^{2}$ ). For decreasing $f$ this can also be obtained by following Zygmund [12, II, p. 130]. Inequality (10.3) can be rewritten as

$$
\mathbf{A}|a|(N) \gg \int_{0}^{1 / N} f(x) d x \quad(N \geqslant 1)
$$

and implies (with absolute constants)

$$
a^{(*)}(N) \gg \int_{0}^{1 / N} f(x) d x \quad(N \geqslant 1) .
$$

Next we can apply Theorem 3 with the intervals shifted by 1 analogously to the proof of (6.1) or (6.2) etc.

THEOREM 7. Assume that $f \in L^{2}[0,1]$ and $V(t)=t^{-2} W(1 / t)$ for $0<t \leqslant 1$. Now we have three cases:

(i) If $0 \leqslant W(t) \searrow(t \geqslant 1)$ and $\Psi(s) \geqslant 0$ is an increasing convex function of $s^{2}$ for $s \geqslant 0$, then

$$
\int_{1}^{\infty} \Psi\left(a^{(*)}\right) W \leqslant \int_{1}^{\infty} \Psi\left(C \int_{0}^{1 / t} f^{*}\right) W=\int_{0}^{1} \Psi\left(C t f^{(*)}(t)\right) V .
$$

(ii) If $0 \leqslant V(t) \nearrow(0<t \leqslant 1)$ and $\Phi(s) \geqslant 0$ is an increasing concave function of $s^{2}$ for $s \geqslant 0$, then

$$
\int_{1}^{\infty} \Phi\left(t a^{(*)}(t)\right) W \leqslant \int_{1}^{\infty} \Phi\left(C t \int_{0}^{1 / t} f^{*}\right) W=\int_{0}^{1} \Phi\left(C f^{(*)}\right) V .
$$

(iii) If $0 \leqslant W(t) \searrow(t \geqslant 1), 1<\alpha<\infty$, and $\Psi(s) \geqslant 0$ is an increasing convex function of $s^{\alpha}$ for $s \geqslant 0$, then

$$
\int_{-\infty}^{\infty} \Psi(|a(s)|) W(1+|s|) d s \geqslant \int_{1}^{\infty} \Psi\left(c_{\alpha} \int_{0}^{1 / t} f\right) W=\int_{0}^{1} \Psi\left(c_{\alpha} \int_{0}^{t} f\right) V
$$


provided that $f \geqslant 0$ on $[0,1]$. ( $C$ is absolute; $c_{\alpha}$ depends on $\alpha$ only.)

The proof of $(10.8)$ reduces to

$$
\int_{|s| \leqslant N-1}|a(s)|^{\alpha} d s \gg \int_{1}^{N}\left(\int_{0}^{1 / t} f\right)^{\alpha} d t \quad(N \geqslant 1)
$$

with a constant depending only on $\alpha$. For $N \in[1,4]$ this can be seen directly while larger $N$ can be treated by (10.4) using Hardy's inequality. The other proofs are as before.

There are equivalent local forms of the inequalties (10.6) and (10.7). All these inequalities are new. The upper estimates and various weaker forms are maximal estimates in terms of $f^{*}$. Of particular interest are the special cases $\Psi(s)=s^{q}$ $(2 \leqslant q<\infty)$ and $\Phi(s)=s^{p}(0<p \leqslant 2)$ of (10.6) and (10.7), respectively. We only mention the local forms

$$
\begin{aligned}
\sum_{n \leqslant N} a_{n}^{(*) q} & \leqslant C^{q} \int_{1 / N}^{1} t^{q-2} f^{(*)}(t)^{q} d t \quad(N \text { real } \geqslant 2), \\
\sum_{n \leqslant N}(n+1)^{p-2} a_{n}^{(*) p} & \leqslant C^{p} \int_{1 / N}^{1} f^{(*)}(t)^{p} d t \quad(N \text { real } \geqslant 2) .
\end{aligned}
$$

These are local versions of the inequalities of Hardy, Littlewood and Pólya. The averaged rearrangements on the right are responsible for the quality of the constant, while the averaged rearrangements on the left are not so essential.

In case $p=1$, we have

$$
\sum_{n \leqslant N} \frac{a_{n}^{*}}{n+1} \leqslant C \int_{0}^{1} f^{*}(t) \min \left(\log \frac{1}{t}, \log N\right) d t \quad(N \geqslant 2)
$$

which, for $N \rightarrow \infty$, turns into

$$
\sum_{n=0}^{\infty} \frac{a_{n}^{*}}{n+1} \leqslant C \int_{0}^{1} f^{*}(t) \log \frac{1}{t} d t .
$$

[Note that the last integral is finite iff $\int_{0}^{1} f^{*} \log ^{+} f^{*}=\int_{0}^{1}|f| \log ^{+}|f|<\infty$.]

By setting $\Phi(t)=t^{p}$ in (10.7) and letting $p \rightarrow+0$ we can infer that (this has the same effect as permitting $\Phi(s)=\log s$, see Hardy, Littlewood and Pólya [5, p. 135, no. 187])

$$
\begin{gathered}
\int_{1}^{N} \log \left(t a^{(*)}(t)\right) \frac{d t}{t^{2}} \leqslant \int_{1 / N}^{1} \log \left(C f^{(*)}(t)\right) d t \quad(N \geqslant 1), \\
\sum_{n \leqslant N} \frac{\log \left[(n+1) a_{n}^{(*)}\right]}{(n+1)^{2}} \leqslant C_{1} \int_{1 / 2 N}^{1} \log \left(f^{(*)}(t)\right) d t+C_{2} \quad(N \geqslant 2),
\end{gathered}
$$

where we assumed $a_{0}^{*} \geqslant 1$ in the last inequality in order to make the indicated simplifications.

Finally we mention that the upper estimates can be extended to orthogonal series, in particular (10.9) and (10.10).

The "global" forms of our inequalities, i.e. limits for $N \rightarrow+\infty$ or $W=1$ in (10.6) and (9.5), or $V=1$ in (10.7) and (9.6) contain, in particular, the known results given 
in Zygmund [12, II, p. 158, 7 and 8]. For the comparison one should make use of our $\S 8$ and consider the influence of $f^{(*)}$ as compared with $f^{*}$, cf. (10.11).

\section{REFERENCES}

1. A. Baernstein, Integral means, univalent functions and circular symmetrization, Acta Math. 133 (1974), 139-169.

2. R. M. Gabriel, A "star inequality" for harmonic functions, Proc. London Math. Soc. 34 (1932), 305-313.

3. G. H. Hardy, Orders of infinity, the "infinitärcalcül" of Paul DuBois-Reymond, Cambridge Univ. Press, London and New York, 1910.

4. G. H. Hardy and J. E. Littlewood, Some new properties of Fourier constants, J. London Math. Soc. 6 (1931), 3-9.

5. G. H. Hardy, J. E. Littlewood and G. Pólya, Inequalities, Cambridge Univ. Press, London and New York, 1934.

6. W. B Jurkat and G. Sampson, On rearrangement and weight inequalities for the Fourier transform, Indiana Math. J. (to appear).

7. J. E. Littlewood, On a theorem of Paley, J. London Math. Soc. 29 (1954), 387-395.

8. On inequalities between $f$ and $f^{*}$, J. London Math. Soc. 35 (1960), 352-365.

9. H. L. Montgomery, A note on rearrangements of Fourier coefficients, Ann. Inst. Fourier (Grenoble) 26 (1976), 29-34.

10. B. Muckenhoupt, Weighted norm inequalities for the Fourier transform, Trans. Amer. Math. Soc. 276 (1983), 729-742.

11. E. M. Stein and G. Weiss, Introduction to Fourier analysis on Euclidean spaces, Princeton Univ. Press, Princeton, N. J., 1971.

12. A. Zygmund, Trigonometric series, Vol. 2, Cambridge Univ. Press, London and New York, 1959.

Department of Mathematics, Syracuse University, Syracuse, New York 13210 (Current address of W. B. Jurkat)

Current address (G. Sampson): Department of Chemistry, Philadelphia College of Textiles \& Science, Philadelphia, Pennsylvania 19144 